\title{
Techniques
}

Acta Cytologica 2012;56:554-559

DOI: $\underline{10.1159 / 000341546}$
Received: March 30, 2012

Accepted after revision: June 28, 2012 Published online: September 27, 2012

\section{Computer-Assisted Diagnosis in Colposcopy: Results of a Preliminary Experiment?}

\author{
Grit Mehlhorn $^{a} \quad$ Christian Münzenmayer $^{\mathrm{b}} \quad$ Michaela Benz $^{\mathrm{b}} \quad$ Andreas Kage $^{\mathrm{b}}$ \\ Matthias W. Beckmann ${ }^{a}$ Thomas Wittenberg ${ }^{\text {b }}$ \\ aDepartment of Gynecology, Erlangen University Hospital, and ${ }^{b}$ Fraunhofer Institute for Integrated Circuits IIS, \\ Erlangen, Germany
}

\section{Key Words}

Cervical intraepithelial neoplasia - Colposcopy ·

Computer-assisted diagnosis - Automated tissue

characterization $\cdot$ Image analysis

\begin{abstract}
Purpose: Diagnosis of cervical intraepithelial neoplasia (CIN) is currently based on the histological result of an aiming biopsy. This preliminary study investigated whether diagnostics for CIN can potentially be improved using semiautomatic colposcopic image analysis. Methods: 198 women with unremarkable or abnormal smears underwent colposcopy examinations. 375 regions of interest (ROIs) were manually marked on digital screen shots of the streaming documentation, which we provided during our colposcopic examinations (39 normal findings, $41 \mathrm{CIN} \mathrm{I}$, and $118 \mathrm{CIN} \mathrm{II-III).} \mathrm{These}$ ROIs were classified into two groups (211 regions with normal findings and CIN I, and 164 regions with CIN II-III). We developed a prototypical computer-assisted diagnostic (CAD) device based on image-processing methods to automatically characterize the color, texture, and granulation of the ROls. Results: Using $n$-fold cross-validation, the CAD system achieved a maximum diagnostic accuracy of $80 \%$ (sensitivity $85 \%$ and specificity $75 \%$ ) corresponding to a correct assignment of abnormal or unremarkable findings. Conclu-
\end{abstract}

\section{KARGER}

Fax +4161306 1234

E-Mail karger@karger.ch

www.karger.com
(C) 2012 S. Karger AG, Basel

0001-5547/12/0565-0554\$38.00/0

Accessible online at:

www.karger.com/acy sions: The CAD system may be able to play a supportive role in the further diagnosis of CIN, potentially paving the way for new and enhanced developments in colposcopy-based diagnosis.

Copyright $\odot 2012$ S. Karger AG, Basel

\section{Introduction}

In Germany, a substantial reduction of the incidence of cervical cancer as well as of the resulting morbidity and mortality could be achieved by the introduction of a cytological public screening system in the early 1970s [1]. The aim of the Pap smear is to identify precancerous lesions in the genitalia as early as possible, clarify them in a differentiated way, and treat them when appropriate. Once an abnormal cytological finding leads us to believe that a precancerous lesion or even a carcinoma could be present, the diagnostic guidelines require a histological analysis to be carried out for confirmation. To enhance the diagnostic accuracy of the assessment, colposcopic examination is an absolute requirement [2-4]. With much-improved visual conditions and the use of various contrast-enhancing solutions (such as acetic acid), better diagnostic clarification is possible on the basis of a targeted biopsy [5]. This type of examination requires exten-

Correspondence to: Dr. Grit Mehlhor

Department of Gynecology, Erlangen University Hospital Universitätsstrasse $21-23$

DE-91054 Erlangen (Germany)

Tel. +4991318533553, E-Mail grit.mehlhorn@uk-erlangen.de 
Fig. 1. Colposcopic nomenclature RIO

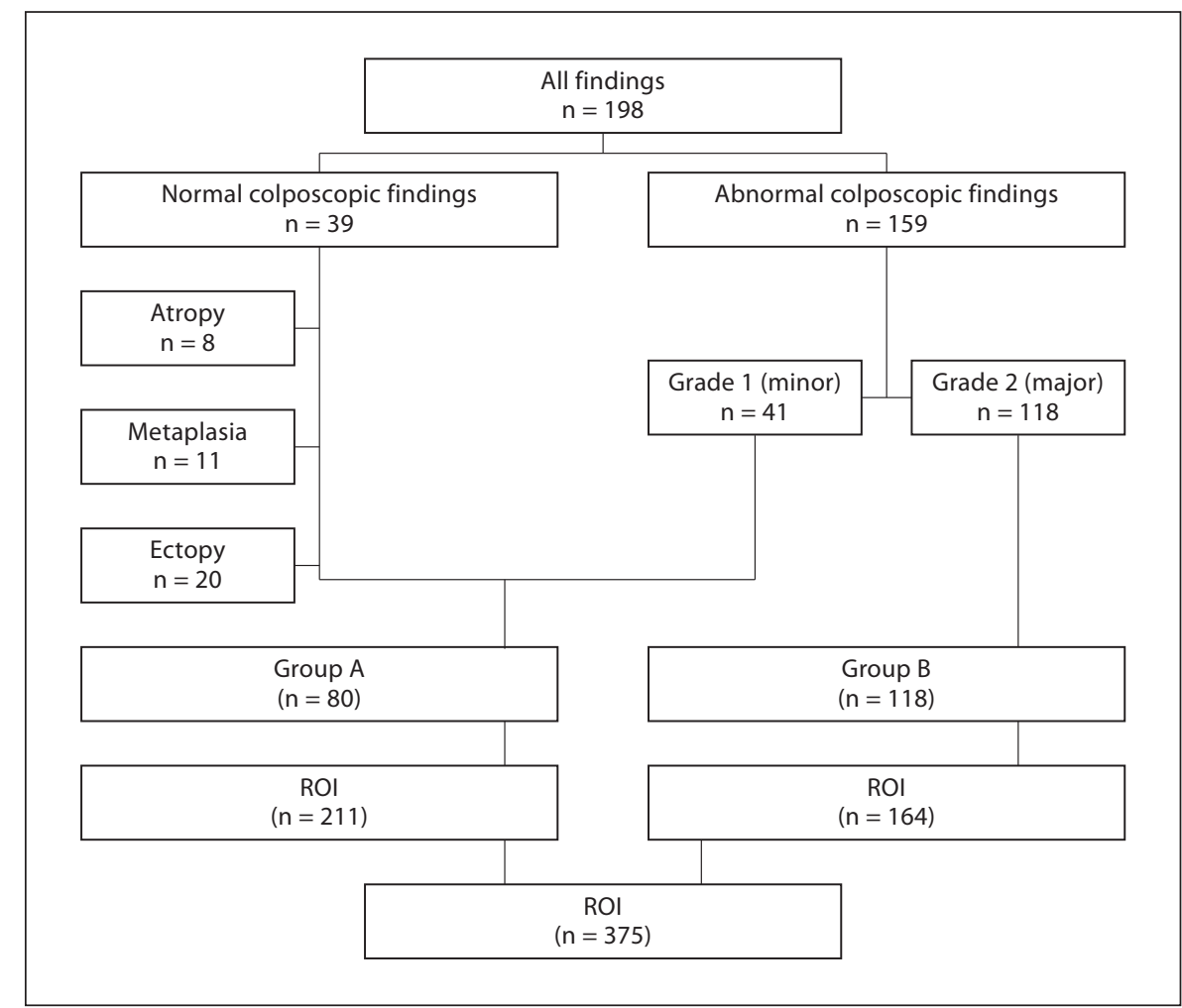
2011.

sive knowledge, and it is also highly dependent on the examiner's objective level of experience [6]. Colposcopy had a sensitivity of $86 \%$ and a specificity of $30 \%$ in distinguishing a healthy cervix from one with cervical intraepithelial neoplasia (CIN)/carcinoma. In distinguishing healthy cervices with low-grade lesions (CIN 1) from those with high-grade lesions (CIN 2/3)/carcinoma, colposcopy had a sensitivity of $61 \%$ and a specificity of $94 \%$ [7]. Despite this, differential colposcopy of this type determines whether a targeted biopsy should be taken and whether histological diagnosis and relevant treatment are carried out $[8,9]$. In addition to the development and evaluation of this type of enhanced assessment method, advances in modern information technology also need to be taken into account. These can provide interactive or automatic support for diagnosis and reporting, and they may also be helpful for training and quality control purposes. Computer-assisted diagnosis (CAD) systems of this sort are already becoming accepted in various medical domains, particularly in preclinical applied research. For example, for the detection of nodules in the lung by computed tomograms [10] and lesions in mammograms [11-13], assessment of endoscopic images in gastroenterology [14-16], or in the field of otorhinolaryngology for analyzing so-called visual biopsies of leukoplakia on the vocal folds [17], preclinical studies have shown that this type of CAD system can be quite helpful for physicians for reporting purposes and for establishing the final diagnosis.

\section{Materials and Methods}

A total of 198 women with either unremarkable or abnormal smears were retrospectively analyzed. The findings were reexamined using a prototypic CAD system for colposcopy developed by the Fraunhofer IIS $[18,19]$. As the automated analysis of colposcopic images is exclusively based on the visual characteristics of the images, only images that were taken with the same magnification level $(\times 0.6)$ and in which the complete vaginal part of the patient's cervix was visible after acetic acid application (fig. 1,2) were analyzed. In addition, the images were selected relative to the focus, contrast and brightness quality of the picture, and in relation to the size and shape of dysplastic changes and their demarcation from the surroundings. The colposcopic criteria for cervical dysplasia in accordance with the new nomenclature had to be appreciable and assignable [5]. Furthermore, as a selection criteria of dysplasia in colposcopic images, the related histological findings had to correspond. During the dysplasia consultation, the internal and external genitalia were initially examined using a cytological smear and colposcopic visualization with acetic acid 
Fig. 2. Green marking of unremarkable findings in the uterine cervix.

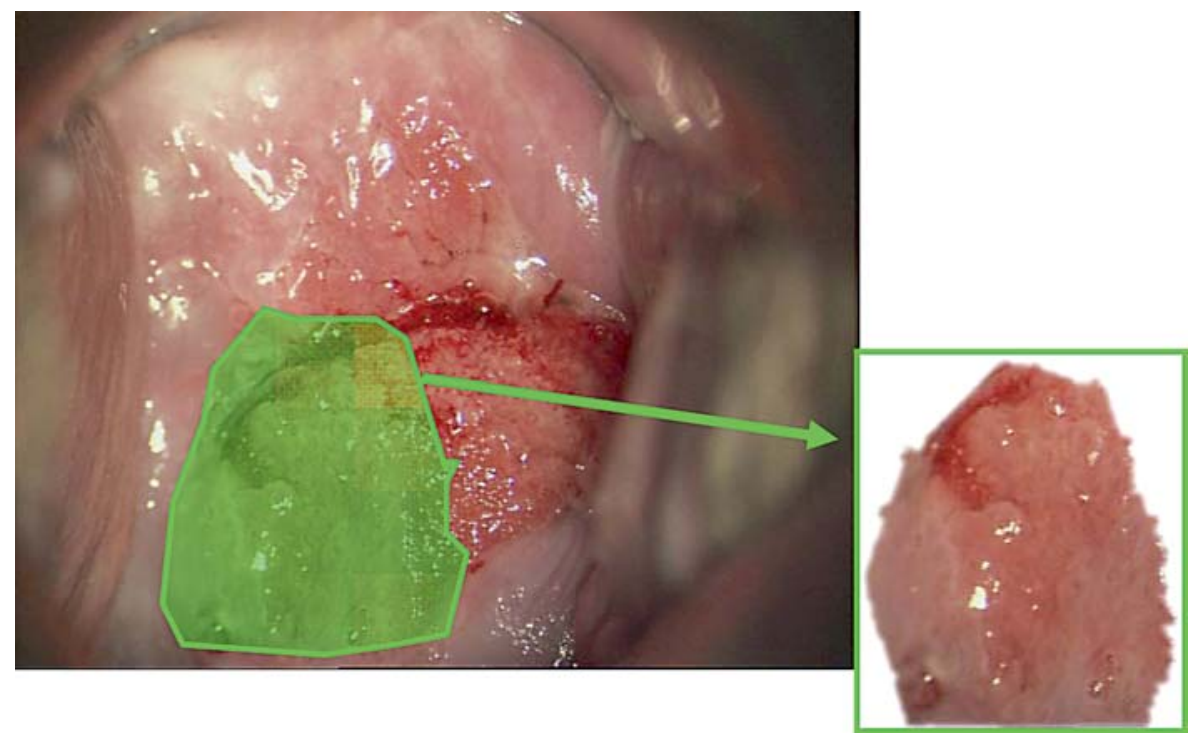

Fig. 3. Red marking of abnormal findings,

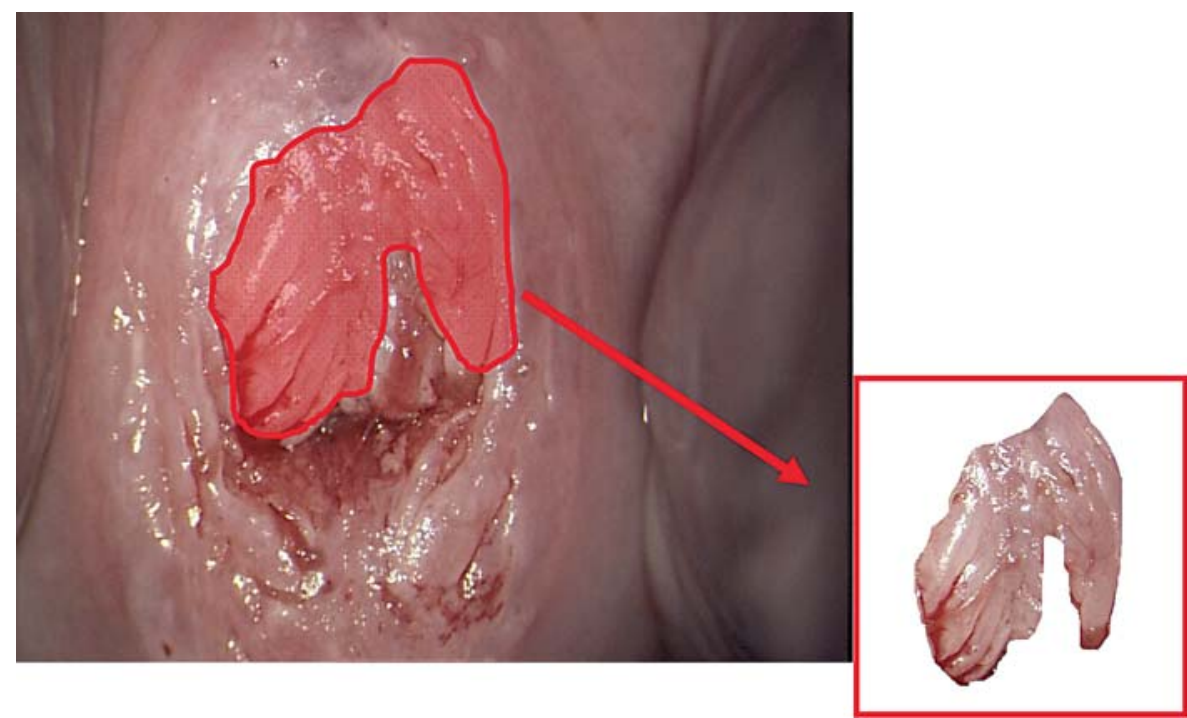
with a CIN III lesion.

(5\%). The capture of the image was taken 1-2 min after application of acetic acid. The colposcopist was accredited by the German Society of Cervical Pathology and Colposcopy. The visual findings were assessed and documented in accordance with RIO 2011 nomenclature, and in case of abnormal findings the relevant histological analysis was carried out.

\section{Image Capture and Image Data}

The visual colposcopic examination was carried out with a binocular colposcope. All images were captured immediately after the acetic acid test using a video camera (Zeiss) mounted on the colposcope, digitized with a frame-grabber card (EYE-2p, RGB), and stored for documentation purposes in the compressed JPEG image format in the ViewPoint image archiving system (GE Healthcare/ViewPoint Bildverarbeitung Ltd., Wessling, Germany). The images for the cases were retrospectively classified into two groups, with one image for each patient. Group A included images from 39 patients with normal findings and 41 patients with low-grade intraepithelial lesions (CIN I). In group B, images from 118 patients with higher-grade intraepithelial lesions were used, corresponding to moderate to severe intraepithelial neoplasia (CIN II and CIN III). An experienced examiner retrospectively outlined and marked 375 representative regions of interest (ROIs) from the collection of 198 colposcopy images and assigned them manually to the two groups using an image annotation tool developed for the study. A total of 211 ROIs (normal/CIN I) in group A and 164 ROIs (CIN II/III) in group B were marked. Higher-grade dysplastic and histologically confirmed lesions (group B) were marked in red and normal or only slightly dysplastic tissue (group A) was marked in green. Figure 1 shows an image with unremarkable findings, while figure 2 illustrates high-grade changes (CIN III) in the cervix. 
Features

Using color texture analysis methods, numerical features were calculated for each of the marked ROIs and used to characterize the marked surface structures on the basis of intensities, structures, textures, and color. The following methods in particular were used $[20,21]$ :

Color histograms (HSTs) show the intensity (or spectral) distributions for all three color channels in an image. In addition to using the three channels from color images in the red, green, and blue (RGB) color space, other color spaces, like for example the Ohta or HSI (hue - saturation - intensity) color spaces [21], can be used to calculate the spectral distributions. To describe the color distributions in an ROI, the HST can be used directly, or representative features such as moments, variances, and energies can be calculated for each sub-histogram.

Color extended statistical geometric features (CESGFs; [22]) describe geometrical micro-structures in an ROI based on statistical features (number, irregularity, mean, and variance) computed from a stack of binary images describing the micro-structures at various levels of intensity. In order to incorporate color into the analysis, the micro-structure statistics are either computed in each color channel alone or correlated with each other during the computation by pairwise combining micro-structures logically.

Color extended gray-level co-occurrence matrices (CEGLCMs) are the most familiar procedures in the field of texture-based tissue characterization. A gray-level co-occurrence matrix (GLCM) describes the frequencies of the co-occurrence of gray-level combinations of the pixels in a certain ROI. From the thus obtained GLCM, a set of 14 features based on statistical characteristics (mean, variance, energy, correlation, entropy, etc.) is computed and used to characterize the underlying tissue. To include color in the computation, the obtained features from the three color channels are fused or, during the calculation of the GLCMs, information from pairwise color channels is combined [21].

Color extended sum and difference histograms (CESDHs) are a fast version of GLCM in which the matrix calculation is approximated using two histograms.

\section{Classification}

To evaluate the CAD system, each of the 375 manually marked image regions (ROIs) was characterized using the groups of features described above (HST, CESGF, CESDH, and CEGLCM) and their variants (resolution reduction, image preprocessing, color correction, and color spaces). On the basis of the feature vectors obtained in this way, a $k$-nearest-neighbor $(\mathrm{kNN})$ classifier [23] with $n$-fold cross-validation was used to evaluate the characteristics of these feature groups relative to the automatic separability of the two groups, i.e. CIN II-III versus CIN I/benign. The kNN classifier selects, for an unknown query vector $q$ with characteristic features, those $k$ vectors in a database of preclassified and histologically validated reference vectors that are most similar to the query vector $q$ relative to a defined distance measure. The diagnostic group and assignment probability of the unknown query vector $q$ are determined on the basis of the vectors most similar to $q$. The kNN has been shown in a large number of studies to be a strong and powerful approach for tasks involving imagingbased distinctions and classifications for medical applications [23].

Computer-Assisted Colposcopy

Diagnosis

\section{Results}

Digital colposcopies for 198 patients, with the 376 manually marked image areas, were used to evaluate the proposed CAD system. The average age of the women examined was 31 years; the youngest patient was 16 and the oldest was 74 years old. The peak age range was $21-42$ years.

Normal findings were noted in 39 of the 80 patients included in group A classified and documented as belonging to the current RIO 2011 colposcopic terminology [24]. Eight patients had atrophic findings, 11 patients had metaplastic findings, and 20 patients had ectopy of the cervix; they were well assessed using colposcopy and were therefore assigned to group A. Forty-one patients in examination group $\mathrm{A}$ had imaging findings with fine acetic acid-positive areas (low-grade CIN). In accordance with the current colposcopic nomenclature, this corresponds to grade 1 abnormal colposcopic findings and is assigned to 'minor changes'. Minor changes on colposcopy involve fine acetic acid-positive findings, with or without a fine punctate or mosaic pattern $[25,26]$. As there is no major difference in treatment implications between this and normal or low-grade lesions, these were assigned to examination group A.

Examination group B included 99 patients with highgrade cervical dysplasia (CIN III) and 19 patients with moderate dysplasia (CIN II). These findings correspond to grade 2 abnormal colposcopic findings in the current colposcopic nomenclature and are therefore assigned to 'major changes'. Major changes describe colposcopically dense acetic acid-positive findings, with or without coarse punctate or coarse mosaic patterns [24]. All dysplastic lesions are histolocically confirmed.

\section{Technical Results}

The diagnostic accuracy currently achievable using the automatic classification of colposcopic lesions - on the basis of the data presented above, in which the CAD system is based on a distinction between high-grade and low-grade lesions - was assessed using what is known as $n$-fold cross-validation in the four groups of features described an their variants. The highest overall classification rate of $80 \%$ (with a sensitivity of $85 \%$ and a specificity of $75 \%$ ) was achieved with CESDHs in combination with image enhancement steps for color correction. The highest specificity of $88 \%$ for recognizing CIN II-III lesions was achieved with HST in the Ohta color space, with an overall classification rate of $75 \%$ and a sensitivity of $64 \%$. A maximum sensitivity (for recognizing CIN I/

Acta Cytologica 2012;56:554-559 
normal tissue) of $75 \%$ was achieved with the statistical geometric features, with an overall classification rate of $78 \%$ and a specificity of $82 \%$.

\section{Discussion}

The CAD system for colposcopic images investigated here shows better accuracy in distinguishing between unremarkable or slightly abnormal cervical findings of very low clinical relevance and higher-grade cervical findings than previous routine diagnosis using a purely visual approach. In indirect comparison with this, Hammes et al. [7] were only able to achieve a maximum sensitivity of $61 \%$ with a specificity of $94 \%$ for enhanced colposcopy in 468 patients with colposcopically evaluated cervical abnormalities when comparing healthy and slightly altered tissue with high-grade and carcinomatous abnormal tissue. Similarly low sensitivity rates were reported by Soutter et al. $[25,26]$. A high sensitivity rate of $86 \%$ for a colposcopic examination to distinguish between healthy and dysplastic cervical epithelium has been reported [7]. Colposcopy during statutory screening for early cancer detection is considered to be clearly inferior to cytology in routine diagnosis. For this reason, colposcopy is only intended for use in diagnostic clarification [24]. The additional use of CAD methods for enhanced colposcopy evidently makes it possible to achieve a marked improvement in the overall accuracy of enhanced diagnosis of clinically relevant precancerous lesions in the uterine cervix. Although these initial results have not been examined further statistically, they can nevertheless be regarded as an improvement on the results reported in the literature with conventional, purely visual colposcopy. In our view, further improvement in the overall recognition rate, sensitivity, and specificity, and in the ability to distinguish between low-grade and high-grade changes in the cervix, would be possible both by standardizing the image capture procedure and by developing specific imaging features. Standardizing the image capture procedure would help improve the CAD approach by producing colposcopic images at a constant image quality, with standard lighting and a standardized image section; and the development and use of new, dedicated features for characterizing tissue could allow the identification of additional differences between highgrade and low-grade lesions. In particular, the potential increase in sensitivity provided by supplementary usage of a CAD system suggests decisive clinical relevance with regard to biopsy clarification and subsequent surgical treatment, as the number of unnecessary biopsies could be reduced. Using digital colposcopy, Schädel et al. [27, 28 ] investigated similar approaches to automated ways of improving diagnostic processes and thereby increasing quality control. CAD of the type evaluated for the first time in the present study on a model basis is not capable of competing with an experienced examiner, far less replacing him or her. However, appropriate CAD systems have tremendous potential in a supportive capacity in the further diagnosis of CIN. CAD systems will not be able to compete with the diagnostic confirmation provided by histology, but they may be able to reduce the need for histological examinations. In principle, this type of CAD system can make it possible in cases of diagnostic uncertainty to use an 'objective' analysis of features in order to systematically improve colposcopy-based diagnosis. The use of standardized imaging techniques to acquire pictures, reproducible cases, and the availability of immediate feedback from the user to the system make the application of this type of CAD system appear feasible both for direct diagnosis and indirectly for training purposes. Further research on such systems is therefore justified. Even though we know that the described experiment is only a preliminary one, based on less than 200 cases, we strongly believe that the first results obtained show the potential of such a system compared to the results cited from the literature. We are currently working toward this end by collecting more data with the same image quality and image resolution, and we are planning such an experiment. Nevertheless, compared to the results in the literature, we believe that our experiments and findings are already showing the tendency and potential to support the assessment of colposcopic images and diagnostic findings.

\section{Disclosure Statement}

There are no conflicts of interest.

References

Mehlhorn/Münzenmayer/Benz/Kage/ Beckmann/Wittenberg screening in Germany. J Cancer Res Clin Oncol 2003;129:691-702.

2 Deutsche Gesellschaft für Gynäkologie und Geburtshilfe: S2k-Leitlinie zur Prävention, Diagnostik und Therapie der HPV-Infektion und präinvasiven Läsionen des weiblichen Genitale - Arbeitsgemeinschaft der Wissenschaftlichen Medizinischen Fachgesellschaften (AWMF), No. 015/027. 2008. http:// leitlinien.net/. 
3 Arbeitsgemeinschaft für Gynäkologische Onkologie: Interdisziplinäre S2k-Leitlinie Diagnostik und Therapie des Zervixkarzinoms. Munich, Zuckschwerdt,2008. http:// www.krebsgesellschaft.de/download/ll_ zervix.pdf.

4 Solomon D, Davey D, Kurman R, et al: The 2001 Bethesda System: terminology for reporting results of cervical cytology. JAMA 2002;287:2114-2119.

5 Nocon M, Mitteldorf T, Roll S, Greiner W, Willich SN, Graf von der Schulenburg JM: Welchen medizinischen und gesundheitsökonomischen Nutzen hat die Kolposkopie als primäres Screening auf das Zervixkarzinom? Cologne, Deutsches Institut für Medizinische Dokumentation und Information, 2007.

6 Jeronimo J, Schiffman M: Colposcopy at a crossroads. Am J Obstet Gynecol 2006;195: 349-353.

7 Hammes LS, Naud P, Passos EP, Matos J, Brouwers K, Rivoire W, Syrjänen KJ: Value of the International Federation for Cervical Pathology and Colposcopy (IFCPC) terminology in predicting cervical disease. J Low Genit Tract Dis 2007;11:158-165.

8 Massad LS, Collins YC: Strength of correlations between colposcopic impression and biopsy histology. Gynecol Oncol 2003;89: 424-428.

-9 Pretorius RG, Bao YP, Belinson JL, Burchette RJ, Smith JS, Qiao YL: Inappropriate gold standard bias in cervical cancer screening studies. Int J Cancer 2007;121:2218-2224.

10 Haider Z, Idris M, Memon WA, Kashif N, Idris S, Sajjad Z, Akram S: Can (CAD) be used as a screening tool in the detection of pulmonary nodules when using 64-slice multidetector computed tomography? Int J Gen Med 2011;4:815-819.

11 Elter M, Schulz-Wendtland R, Wittenberg T: The prediction of breast cancer biopsy outcomes using two CAD approaches that both emphasize an intelligible decision process. Med Phys 2007;34:4164-4172.

- 12 Romero C, Varela C, Muñoz E, Almenar A, Pinto JM, Botella M: Impact on breast cancer diagnosis in a multidisciplinary unit after the incorporation of mammography digitalization and computer-aided detection systems. AJR Am J Roentgenol 2011;197:14921497 (erratum published in AJR Am J Roentgenol 2012;198:2).
13 Moin P, Deshpande R, Sayre J, Messer E, Gupte S, Romsdahl H, Hasegawa A, Liu BJ: An observer study for a computer-aided reading protocol (CARP) in the screening environment for digital mammography. Acad Radiol 2011;18:1420-1429.

14 Münzenmayer C, Kage A, Wittenberg T, Mühldorfer S: Computer-assisted diagnosis for precancerous lesion in the esophagus. Methods Inf Med 2009;48:324-330.

15 Zopf S, Kage A, Münzenmayer C, Wittenberg T, Hahn EG, Raithel M: Narrow-band imaging for computer-assisted diagnosis in patients with Barrett's esophagus (abstract). Gastrointest Endosc 2009;69:AB376.

16 Rajan P, Canto M, Gorospe E, Almario A, Kage A, Winter C, Hager, Wittenberg T, Münzenmayer C: Automated diagnosis of Barrett's esophagus with endoscopic images; in Dössel O, Schlegel WC (eds): World Congress on Medical Physics and Biomedical Engineering: 7-12 September, 2009, Munich, Germany. International Federation for Medical and Biological Engineering: proceedings, vol 25, part 4: Image processing, biosignal processing, modelling and simulation, biomechanics. Berlin, Springer, 2009, pp 2189-2192.

17 Kothe C, Münzenmayer C, Wittenberg T, Hess M: Experiences with 'optical' biopsies of leukoplakia of the vocal folds (in German). Laryngorhinootologie 2005;84:92-95.

18 Kage A, Münzenmayer C, Wittenberg T: A knowledge-based system for the computer assisted diagnosis of endoscopic images; in Tolxdorff T, Braun J, Deserno TM, Handels $\mathrm{H}$, Horsch A, Meinzer HP (eds): Bildverarbeitung für die Medizin 2008. Algorithmen - Systeme - Anwendungen. Proceedings des Workshops vom 6. bis 8. April 2008 in Berlin. Springer, Berlin, 2008, pp 272-276.

19 Wittenberg T, Kage A, Benz B, Winter C, Koch M, Beckmann M, Münzenmayer C, Mehlhorn G: GynCAD - a computer-assisted diagnosis approach for colposcopy; in Horsch A, et al (eds): Abstracts - Demonstration Workshop on Computer-Aided Detection and Diagnosis, CARS-Workshop, June 23/26, 2010, Geneva, 2010, pp 7-8.

20 Münzenmayer C, Mühldorfer S, Mayinger B, Volk H, Grobe M, Wittenberg T: Farbtexturbasierte optische Biopsie auf hochauflösenden endoskopischen Farbbildern des Ösophagus; in Wittenberg T, et al (eds): Bildverarbeitung für die Medizin 2003. Algorithmen - Systeme - Anwendungen. Proceedings des Workshops vom 9.-11. März 2003 in Erlangen. Springer, Berlin, 2003, pp 191-195.
21 Palm C, Metzler V, Mohan B, Dieker O, Lehmann T, Spitzer K: Co-Occurrence Matrizen zur Texturklassifikation in Vektorbildern; in Evers H, et al (eds) Bildverarbeitung für die Medizin 1999. Algorithmen - Systeme - Anwendungen. Proceedings des Workshops am 4. und 5. März 1999 in Heidelberg. Springer, Berlin, 1999, pp 367-371.

22 Münzenmayer C, Volk H, Paulus D, Vogt F, Wittenberg T: Multispectral statistical geometrical features for texture analysis and classification; in Franke KH (ed): Proceedings 8. Farbbildverarbeitung, Zentrum für Bild- und Signalverarbeitung e.V. Ilmenau, Workshop, 10./11.10.2002, TU Ilmenau, 2002, pp 87-94.

23 Michie D, Spiegelhalter DJ, Taylor CC: Machine Learning, Neural and Statistical Classification. New York, Ellis Horwood, 1994.

24 Bornstein J, Bentley J, Bosze P, Girardi F, Haefner H, Menton M, Perrotta M, Prendiville W, Russell P, Sideri M, Strander B, Torne A, Walker P: IFCPC colposcopic nomenclature. In preparation.

25 Soutter WP, Diakomanolis E, Lyons D, Ghaem-Maghami S, Ajala T, Haidopoulos D, Doumplis D, Kalpaktsoglou C, Sakellaropoulos G, Soliman S, Perryman K, Hird V, Buckley CH, Pavlakis K, Markaki S, Dina R, Healy V, Balas C: Dynamic spectral imaging: improving colposcopy. Clin Cancer Res 2009; 15:1814-1820.

26 Ferris DG, Litaker MS, Miller JA, Macfee MS, Crawley D, Watson D: Qualitative assessment of telemedicine network and computer-based telecolposcopy. J Low Genit Tract Dis 2002;6:145-149.

-27 Schädel D, Coumbos A, Willrodt RG, Roggan A, Jochum T, Müller G, Albrecht $\mathrm{H}$, Kühn W: Digitale Kolposkopie bei Läsionen der Cervix uteri - eine Pilotstudie unter Berücksichtigung telematischer Fragestellungen. Geburtshilfe Frauenheilkd 2004;64: 1205-1212.

28 Schädel D, Coumbos A, Drechsler I, Ey S, Weissbach C, Albrecht H, Lochmann C, Kuehn W: New research on colposcopy: results of a two-phase study to test digital colposcopy and telecolposcopy in clinical practice. J Turk Ger Gynecol Assoc 2006;7:282291. 